\section{BRAZIULIAN JOURNAL \\ OF MEDICAL AND BIOLOGICAL RESEARCH}

www.bjournal.com.br
ISSN 1414-431X

Volume 45 (12) 1102-1340 December 2012

BIOMIDICAL SCIENCES

AND

CLINICAL INVESTIGATION

Braz J Med Biol Res, December 2012, Volume 45(12) 1221-1233

doi: 10.1590/S0100-879X2012007500137

Relationships between dendritic morphology, spatial distribution and firing patterns in rat layer 1 neurons

D.V.V. Santos, K.M. Costa, M.C.G. Vaz and M. Da Silva Filho

The Brazilian Journal of Medical and Biological Research is partially financed by

\section{욛NPq}

Ministério

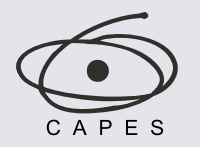

Ministério da Educação

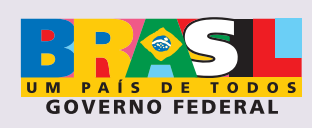

DTFAPESP

Institutional Sponsors

๑ SHIMADZu

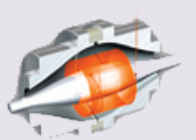

М디맘

Associaçäo
Fundo

Explore High - Performance MS Orbitrap Technology

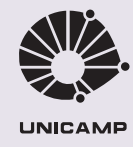
analitica Thermo 


\title{
Relationships between dendritic morphology, spatial distribution and firing patterns in rat layer 1 neurons
}

\author{
D.V.V. Santos, K.M. Costa, M.C.G. Vaz and M. Da Silva Filho \\ Laboratório de Biofísica Celular, Instituto de Ciências Biológicas, \\ Universidade Federal do Pará, Belém, PA, Brasil
}

\begin{abstract}
The cortical layer 1 contains mainly small interneurons, which have traditionally been classified according to their axonal morphology. The dendritic morphology of these cells, however, has received little attention and remains ill defined. Very little is known about how the dendritic morphology and spatial distribution of these cells may relate to functional neuronal properties. We used biocytin labeling and whole cell patch clamp recordings, associated with digital reconstruction and quantitative morphological analysis, to assess correlations between dendritic morphology, spatial distribution and membrane properties of rat layer 1 neurons. A total of 106 cells were recorded, labeled and subjected to morphological analysis. Based on the quantitative patterns of their dendritic arbor, cells were divided into four major morphotypes: horizontal, radial, ascendant, and descendant cells. Descendant cells exhibited a highly distinct spatial distribution in relation to other morphotypes, suggesting that they may have a distinct function in these cortical circuits. A significant difference was also found in the distribution of firing patterns between each morphotype and between the neuronal populations of each sublayer. Passive membrane properties were, however, statistically homogeneous among all subgroups. We speculate that the differences observed in active membrane properties might be related to differences in the synaptic input of specific types of afferent fibers and to differences in the computational roles of each morphotype in layer 1 circuits. Our findings provide new insights into dendritic morphology and neuronal spatial distribution in layer 1 circuits, indicating that variations in these properties may be correlated with distinct physiological functions.
\end{abstract}

Key words: Biocytin; Patch-clamp; Dendritic morphology; Layer 1; Neuronal variability

\section{Introduction}

Cortical layer 1 is composed mainly of small, nonpyramidal, GABAergic neurons (1-3). These cells typically receive input from dense axonal fibers of intracortical and subcortical origin, and their own axons synapse onto the apical dendrites of pyramidal cells and other layer 1 neurons $(1,3,4)$.

There are many hypotheses concerning the physiological role of layer 1 neurons. Vogt (1) argued that one of the essential functions of layer 1 circuits is event holding, similarly to a "sample and hold" function of electronic circuits. It has also been proposed that these cells may provide feedfoward inhibition with incoming information, thus modulating the response of underlying cortical circuits by direct inhibition or disinhibition (5). Other models suggest that these networks may integrate intracortical and thalamical input in a coherent, time-sensitive manner (6). Another proposed function is that layer 1 interneurons provide tonic inhibition to underlying cortical layers in physiological conditions, thus playing an important role in determining the degree of general excitability of the neocortex (4). Recently, Zhu and Zhu (7), using in vivo intracellular recordings and applying realistic sensory stimulation protocols, concluded that layer 1 circuits may also selectively amplify salient sensory input that arrives at different cortical layers.

Which of these complex mechanisms (or if, to some extent, all of them) represent the main function of this cortical layer is a highly debated issue. There is also very little conclusive information about the dynamics of local layer 1 circuits. With the exception of Cajal-Retzius cells, which play a critical role in the development of the mammalian

Correspondence: D.V.V. Santos, Laboratório de Biofísica Celular, Instituto de Ciências Biológicas, Universidade Federal do Pará, Rua Augusto Corrêa, 1, 66075-110 Belém, PA, Brasil. E-mail: danielsantos@ufpa.br

Received January 12, 2012. Accepted August 15, 2012. Available online August 31, 2012. Published December 17, $2012 .$. 
cortex $(2,8)$, the specific function of other classes of layer 1 neurons remains ill defined.

The lack of a comprehensive, quantitative classification of these cells is still a major hindrance for evaluating the functional roles of layer 1 interneurons. Previous efforts by Zhou and Hablitz $(2,5,9,10)$ and Hestrin and Armstrong (11), in spite of providing crucial insights into the general morphology, membrane properties, synaptic physiology, and development of layer 1 neurons, have failed to produce a robust morphophysiological categorization of these cells, in large part due to the low number of studied neurons and the use of purely qualitative morphological analysis.

Traditionally, cortical interneurons have been classified according to their axonal arbor (12), leading to the mainly qualitative segregation of these cells into groups such as neurogliaform cells (small cells with dense axonal arborization) or chandelier cells (neurons whose axonal clusters are highly ramified and at shallow angles) (12). The dendritic morphological properties of these neurons have, however, been conspicuously overlooked, in great part due to the notion that the interneuron dendritic arbor is so variable that no significant correlation can be obtained by studying it (12). Nonetheless, there is surmounting evidence that the computational processes executed by single neurons are mainly dependent on their dendritic properties (13-15), which makes dendritic morphology a crucial parameter in understanding how neurons integrate synaptic input.

The aim of the present study was to evaluate the possible relationships between the dendritic morphology, spatial distribution and electrophysiological characteristics of layer 1 interneurons of the rat neocortex. In order to accomplish this goal, we used whole cell patch-clamp recordings associated with biocytin staining followed by the digital reconstruction of the dendritic morphology of labeled neurons. Our main hypothesis was that neuronal populations with different dendritic properties may serve distinct functions in the layer 1 circuitry, which could in turn be associated with specific firing patterns or other electrophysiological characteristics. We, therefore, categorized the recorded and labeled cells according to the quantitative morphological parameters of their dendritic processes, their distribution in the sublayer strata of layer 1 and their firing patterns. We then determined if there was any statistical correlation between their morphological (morphotype and spatial distribution) and electrophysiological (active and passive membrane properties) characteristics. Our findings shed new light on the relationship between dendritic morphology and the physiological function of cortical interneurons and provide a novel background for the study on the overall functions of layer 1.

\section{Material and Methods}

\section{Brain slice preparation \\ All experiments were carried out in accordance with the}

National Institute of Health Guide for the Care and Use of Laboratory Animals (NIH Publications No. 80-23, revised 1996), and were approved by the Ethics Research Committee for Animal Experiments of the Instituto de Ciências Biológicas, Universidade Federal do Pará.

Slices of rat primary visual cortex (area 17) were prepared according to the procedures described by Edwards et al. (16). In brief, young Wistar rats (14-21 days of postnatal life) were decapitated, and the brain was rapidly removed from the skull and immersed in ice-cold artificial cerebrospinal fluid (ACSF). Brain slices were cut on a vibratome (Pelco, USA) at $250 \mu \mathrm{m}$ thickness and stored at room temperature $\left(23^{\circ} \mathrm{C}\right)$ for $1 \mathrm{~h}$ in ACSF constantly bubbled with a carbogenic mixture $\left(95 \% \mathrm{O}_{2}, 5 \% \mathrm{CO}_{2}\right)$ before the recording procedures. Individual slices were placed in a recording chamber mounted on the stage of a Zeiss Axioskop 2 FS Plus microscope (Carl Zeiss AG, Germany). The recording chamber was constantly perfused with ACSF at a rate of $4 \mathrm{~mL} / \mathrm{min}$, resulting in a complete solution exchange every $5 \mathrm{~min}$. The ACSF bath solution contained $125 \mathrm{mM}$ $\mathrm{NaCl}, 3.5 \mathrm{mM} \mathrm{KCl}, 2 \mathrm{mM} \mathrm{CaCl}_{2}, 1.3 \mathrm{mM} \mathrm{MgCl}_{2}, 26 \mathrm{mM}$ $\mathrm{NaHCO}_{3}$, and $10 \mathrm{mM}$ D-glucose. The perfusing solution was also continuously bubbled with $95 \% \mathrm{O}_{2}$ and $5 \% \mathrm{CO}_{2}$ to maintain the $\mathrm{pH}$ at 7.4 .

\section{Electrophysiological recordings}

Individual cells were visualized using a 40-X water immersion objective with 3.3-mm working distance (Carl Zeiss AG) and Nomarski optics. Layer 1 neurons were located using the pial surface and the border between layer 1 and layer $2 / 3$ as reference points. Cells were recorded and filled across the entire thickness of the layer.

Patch recording electrodes made of borosilicate glass (BF150-110-10, Sutter Instrument Co., USA) and with a 4-5 $\mathrm{M} \Omega$ resistance in bathing solution were used for intracellular recordings. The micropipettes were filled with a solution containing $125 \mathrm{mM}$ K-gluconate, $10 \mathrm{mM} \mathrm{KCl}, 10 \mathrm{mM} \mathrm{N}$ hydroxyethyl-piperazine-N'-2-ethanesulfonic acid, $2 \mathrm{mM}$ Mg-ATP, $0.2 \mathrm{mM} \mathrm{Na-GTP,} \mathrm{and} 0.5 \mathrm{mM}$ ethylene glycol-bis ( $\beta$-aminoethyl ether) N,N,N',N'-tetraacetic acid. Biocytin (Sigma-Aldrich, USA) at $0.6 \%$ was also included in the patch electrode filling solution and allowed to diffuse into the cell. The liquid junction potential, which averaged $8.53 \mathrm{mV}$, was automatically calculated and corrected using pClamp 10.2 software applications before each analysis.

Seal resistance was always $>5 \mathrm{G} \Omega$ before breaking into whole cell mode. The resting membrane potential (RMP) was measured immediately after this event. The effects of membrane potential on the firing properties of layer 1 neurons were evaluated by evoking action potentials with depolarizing current pulses (10 to $100 \mathrm{pA}$ ) while cells were held at the membrane potential of $-100 \mathrm{mV}$. Input resistance $\left(R_{\text {in }}\right)$ was measured by injecting small hyperpolarizing current pulses (-10 to $-100 \mathrm{pA})$.

Analog voltage signals were recorded in current-clamp 
mode using a Multiclamp 700 B amplifier (Molecular Devices, USA). Digital data acquisition and analysis were performed using the pClamp 10.2 software (Molecular Devices). At the end of the each recording, cells were maintained at $-65 \mathrm{mV}$ for $10 \mathrm{~min}$ in order to increase biocytin transport into remote processes. After this procedure the slices were transferred to $4 \%$ paraformaldehyde in $0.1 \mathrm{M}$ phosphatebuffered saline, $\mathrm{pH} 7.5$, and fixed for $24-48 \mathrm{~h}$ at $4^{\circ} \mathrm{C}$.

\section{Histological processing}

Tissue samples were processed as described by Horikawa and Armstrong (17). In brief, slices were rinsed in $0.1 \mathrm{M} \mathrm{Na}$-phosphate buffer and then treated with $1 \% \mathrm{H}_{2} \mathrm{O}_{2}$ for 2-3 $\mathrm{h}$ at room temperature. Next, they were incubated for $12 \mathrm{~h}$ at $4{ }^{\circ} \mathrm{C}$ in blocking serum containing $2 \%$ bovine serum, $1 \%$ bovine serum albumin, and $0.4 \%$ Triton $\mathrm{X}-100$. After rinsing, the sections were incubated with the avidinbiotin-peroxidase complex (Vectastain Elite ABC kit; 1:100; Vector Laboratories, USA) in $1 \%$ bovine serum albumin for $4 \mathrm{~h}$. Sections were rinsed, stained with 3,3-diaminobenzidine, mounted on gelatin-coated glass slides, dehydrated, and covered with a coverslip. The Nissl method was used for counterstaining.

\section{Data analysis}

The membrane time constant $(\tau)$ was calculated according to the method proposed by Rall (15). In brief, we applied Equation 1 using the pClamp 10.2 software.

$$
f(t)=\sum_{i=1}^{n} A_{i} e^{-t / \tau_{i}}+C
$$

In this equation, $t$ represents time, $C$ represents the steadystate asymptote, $A_{i}$ represents the relative amplitude at the start of the fit region, and $e$ is the Euler number.

After-potentials were then identified and measured according to the definition of Storm (18). According to this study, hyperpolarizations can be subdivided into fast (fAHP), slow (sAHP) and medium (mAHP) according to their kinetics and underlying currents. fAHPs last about 2-5 ms and are followed by repolarization; mAHPs last between 50 and $100 \mathrm{~ms}$ and sAHPs last about 1-2 s. In our experiments, all AHPs were measured after a train of action potentials. fAHPs, mAHPs, and sAHPs were measured in relation to the membrane threshold, i.e., the membrane potential immediately prior to the initiation of the action potential. This threshold represents the membrane potential where there is a maximum opening probability of voltage-gated sodium channels for the generation of an action potential and is classically used for calculating the amplitude of afterpotentials (2). After-depolarization potentials (ADPs) were measured in relation to the peak of the preceding fAHP (2). The remaining active and passive electrophysiological properties were analyzed using computer-generated commands in the Clampfit 10.2 software interface.

According to Vogt (1), layer 1 can be divided into three sub-laminae according to physiological and anatomical characteristics, including the origin and distribution of input fibers. In our study, layer 1 neurons were grouped according to their relative distance from the inferior pial surface and the limit between layer 1 and layer 2/3 (Figure 1). This was done in order to assess possible correlations between morphophysiological parameters and spatial distribution of layer 1 cells. Neurons located in the upper third of the layer were classified as "superior cells", those positioned in the middle third were classified as "medial cells", and those in the lower third were classified as "inferior cells". The proportional distance from the reference points was calculated based on the local thickness of layer 1 for each cell. The limits of each sub-layer were determined separately for each cell while the proportion ratio was always kept constant, so as to not generate any bias related to tissue remodeling induced by histological processing.

Biocytin-labeled neurons were photographed with a Zeiss Axiophot camera (Carl Zeiss AG) in 2- $\mu$ m discrete steps along the Z-axis. Individual focal planes were merged and digital reconstructions of the cells were made using the Neuron J plug-in for ImageJ (19). Some representative cells were also drawn by hand using a camera lucida, for expositional purposes. The dendritic orientation of individual neurons and the compound morphological groups were plotted as polar histograms using a customized script for Matlab R2008a (Mathworks, USA). In brief, the surrounding area of the soma was defined as a circle centered in the middle of the soma and divided into $15^{\circ}$ arches. The length of the dendrites confined to each arch was measured using Neuron J. The arches were then grouped into two $100^{\circ}$ arches above and below the soma and two $80^{\circ}$ arches laterally to the soma and the total dendritic length confined

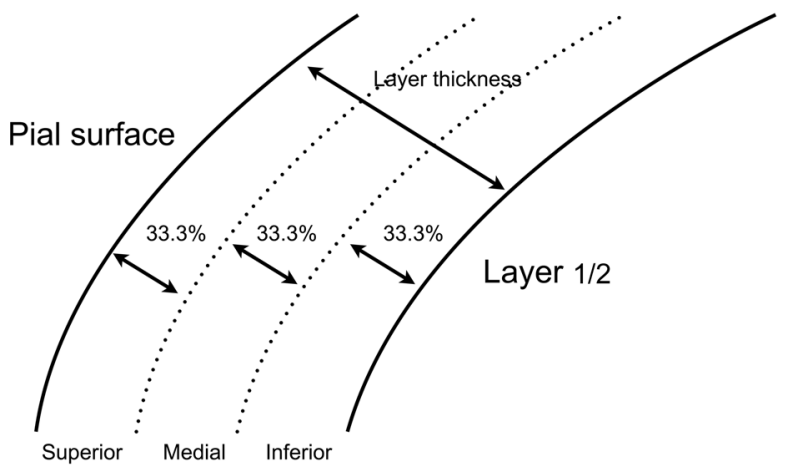

Figure 1. Schematic presentation of parameters used for spatial distribution analysis in the present study. Three equally sized sublayers were defined according to their relative size (33.3\%) in relation to the total thickness of layer 1. Labeled neurons were classified according to their location in any of the three sub-layers. 
to each arch group was quantified and plotted in the graph. Morphotypical classifications were then made according to the distribution of dendritic clusters in the aforementioned arches. Similar analytical methods have been described by Hamam et al. (20) and Romanova et al. (21).

Ramification patterns of the dendritic arbors were evaluated with the Sholl analysis method $(22,23)$ using the Sholl Analysis plug-in for ImageJ (24). In brief, the software generates a series of concentric circles centered at the neuron's soma in such a manner that each circle has a radius that is $5 \mu \mathrm{m}$ greater than its predecessor in the series. The software then superimposes the circles on the image of the cell and counts how many times the neuronal processes intersect with the circumference of each circle. General morphological parameters such as the total dendritic length were evaluated with the aid of the Neuron J plug-in.

All quantitative results, unless noted, are reported as means \pm SEM. For continuous numerical variables, such as the passive membrane properties, statistical comparisons were made using Kruskal-Wallis ANOVA $(\alpha=0.05)$. Differences in the statistical distribution of qualitative variables, such as the proportion of distinct firing patterns, were

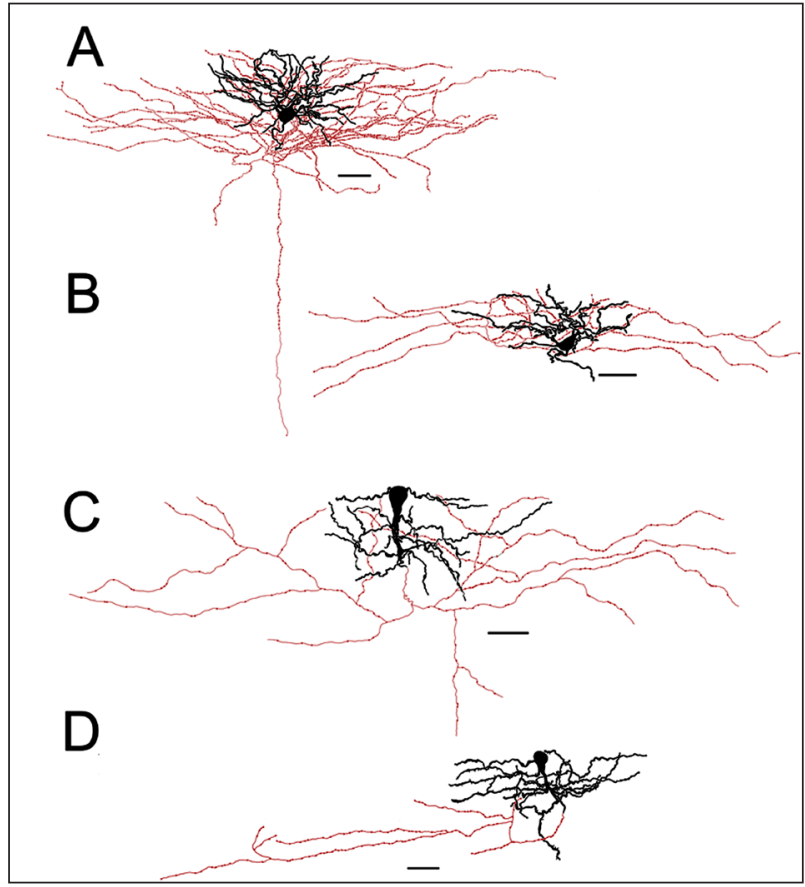

Figure 2. Examples of camera-lucida drawings of biocytinlabeled layer 1 cells. $A, B$, Ascendant cells, defined by their dendritic projections (black), which extend upwards in relation to the cell body. The neuron in $A$ exhibited a d-NAC firing pattern, while the neuron in $B$ exhibited a $C-A C$ firing pattern. $C$, $D$, Descendant cells, defined by their dendritic arbor, which projects downwards from the cell body. Axon arborization (red) was not evaluated in this study. Scale bar $=50 \mu \mathrm{m}$. d-NAC $=$ delayed non-accommodating cell; c-AC = classic accommodating cell. evaluated with the G-test, a more powerful and accurate version of the chi-square test $(\alpha=0.05)$.

\section{Results}

A total of 244 cells were recorded, of which 106 were successfully labeled and recovered for morphological analysis. The recovered cells were divided into four major morphotypes according to the quantitative orientation of their dendritic arbors (Figures 2 and 3). Dendritic field orientation was the main parameter for morphological segregation, as it is tightly related to the nature of the neuron's synaptic input $(1,14,20)$. Neuronal morphotypes were established according to the distribution of dendritic clusters in a polar plot (Figure 4). The established morphotypes were: horizontal cells $(26.4 \%$ of total cells; $28 / 106)$, defined by having over $50 \%$ of their dendritic processes concentrated in an $80^{\circ}$ arch lateral to the soma; radial cells (38.7\%; 41/106), which were neurons where the dendritic cluster distribution did not show a preferential distribution, i.e., their dendritic clusters

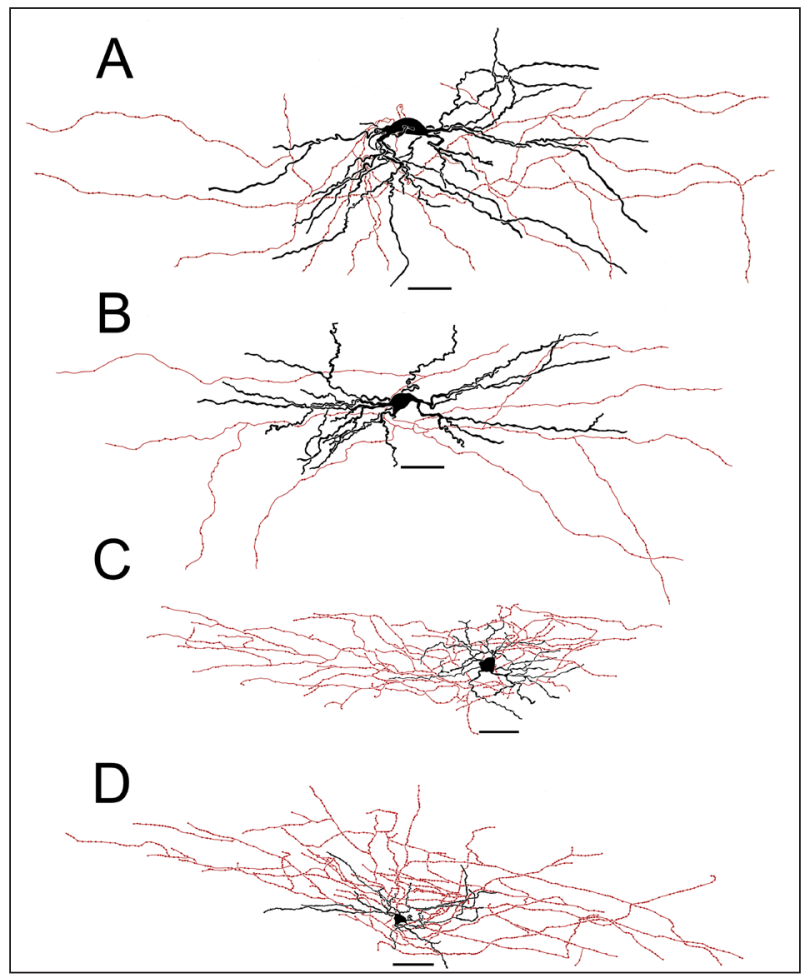

Figure 3. Examples of camera lucida drawings of biocytin-labeled layer 1 cells. $A, B$, Horizontal cells, defined by their dendritic arbors (black), which project horizontally from the cell body. The neuron in $A$ exhibited a c-NAC firing pattern, while the neuron in $B$ exhibited a c-STUT firing pattern. $C, D$, Radial cells, defined by their irregular dendritic arbor in all directions. The neuron in $C$ exhibited a C-NAC firing pattern, while the neuron in $D$ exhibited a c-AC firing pattern. Axon arbors (red) were not evaluated in this study. Scale bar $=50 \mu \mathrm{m}$. c-NAC $=$ classic non-accommodating cell; c-STUT $=$ classic stuttering cell. 

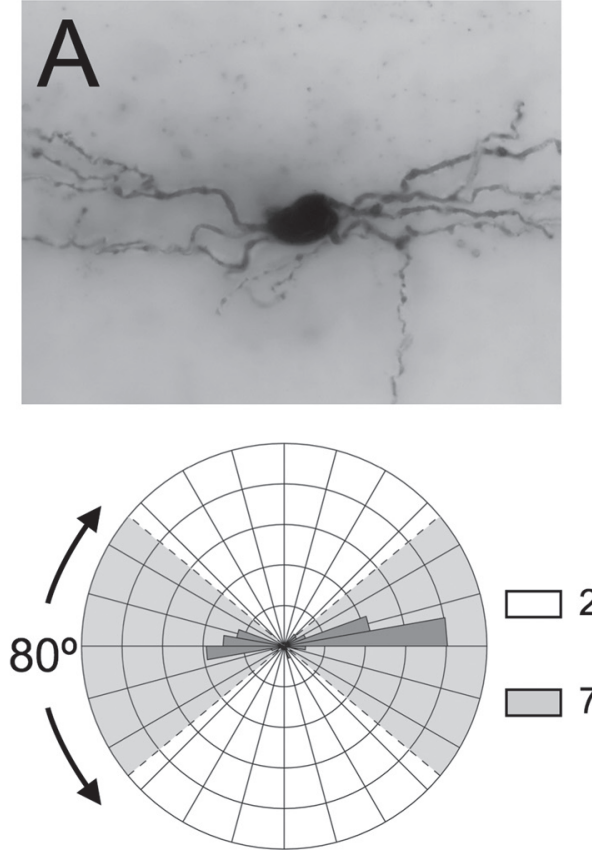

$29.5 \%$

$70.5 \%$
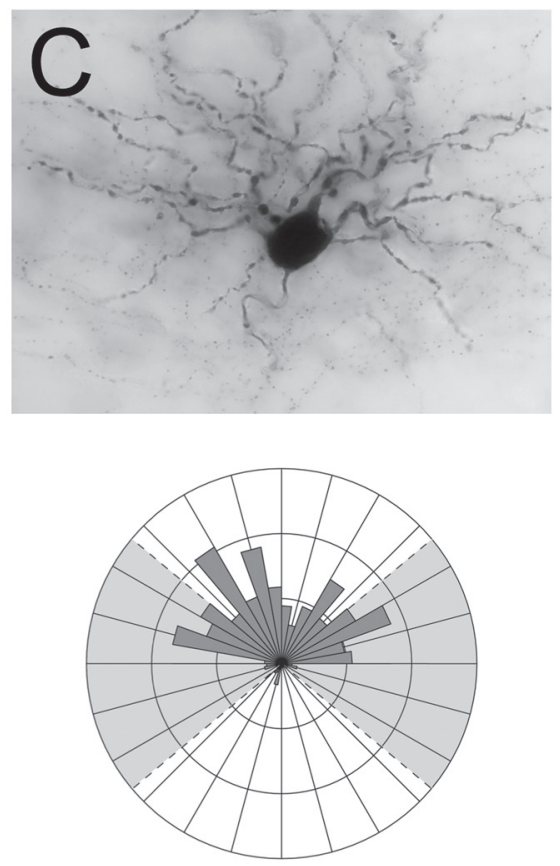

Figure 4. Morphological subtypes of labeled layer 1 neurons. $A-D$, Micrographs of representative cells for each subtype (horizontal, descendant, ascendant, and radial, respectively), alongside their respective representative polar histograms. The neuron in A exhibited a c-NAC firing pattern, the neuron in $C$ exhibited a d-NAC firing pattern and the neuron in $D$ exhibited a c-NAC firing pattern. Legends indicate the proportion of the total dendritic length of the compound population of each morphological subtype that occupies the referenced area. Note that for the descendant cells, half of the total dendritic length is concentrated in the area delimited by a $100^{\circ}$ angle directly underneath the soma, indicating a marked downward distribution. Scale bar $=20 \mu \mathrm{m}$ for all cells. c-NAC = classic non-accommodating cell; $\mathrm{d}-\mathrm{NAC}=$ delayed non-accommodating cell. 
did not show a $50 \%$ overlap with any of the established analytical regions; ascendant cells (20.8\%; 22/106), which had over $50 \%$ of their dendritic arbors projecting above to the region directly above the soma, and descendant cells (12.3\% of the total cells analyzed; $13 / 106)$, whose dendrites projected mainly directly below the soma (over $70 \%$ ). A few cells $(1.8 \% ; 2 / 106)$ were too irregular to be classified into the established morphotypes and were left unclassified.

About 25\% (7/28) of horizontal cells had dendritic clusters that projected mainly unilaterally and 75\% (21/28) bilaterally. They also had, in general, an ellipsoid and small soma. Radial cells had a round or irregular cell body; ascendant cells usually had round or ellipsoid cell bodies, and descendant cells had an irregular or elongated cell body. Among descendant cells, a particular subtype can be distinguished, i.e., the subpial cells. These cells, also called "vertical cells" by Bradford et al. (25) and Zhou and
Hablitz (5), represented $76.9 \%$ (10/13) of the descendant cell population. They were located only immediately under the pial border, possessed particularly long cell bodies and had a singular pattern of dendritic ramification, with a large number of dendrites being emitted laterally and downwards from the cell body. Even though these cells may be a distinct subtype on their own, because of their relative scarcity in our total sample size (10 of 106 cells) they were grouped with other descendant cells for all analytical purposes.

Sholl analysis revealed that all layer 1 neurons achieved their peak branching at about 25-30 $\mu \mathrm{m}$ of distance from the soma (Figure 5A). Horizontal, radial and ascendant cells had branching peaks of similar value, i.e., approximately 9-10 ramifications. Descendant cells, however, exhibited a significantly smaller peak in ramification $(6.3 \pm 0.89$ intersections; $P=0.0107)$. We found significant differences in the number of intersections per $5 \mu \mathrm{m}$, total dendritic length
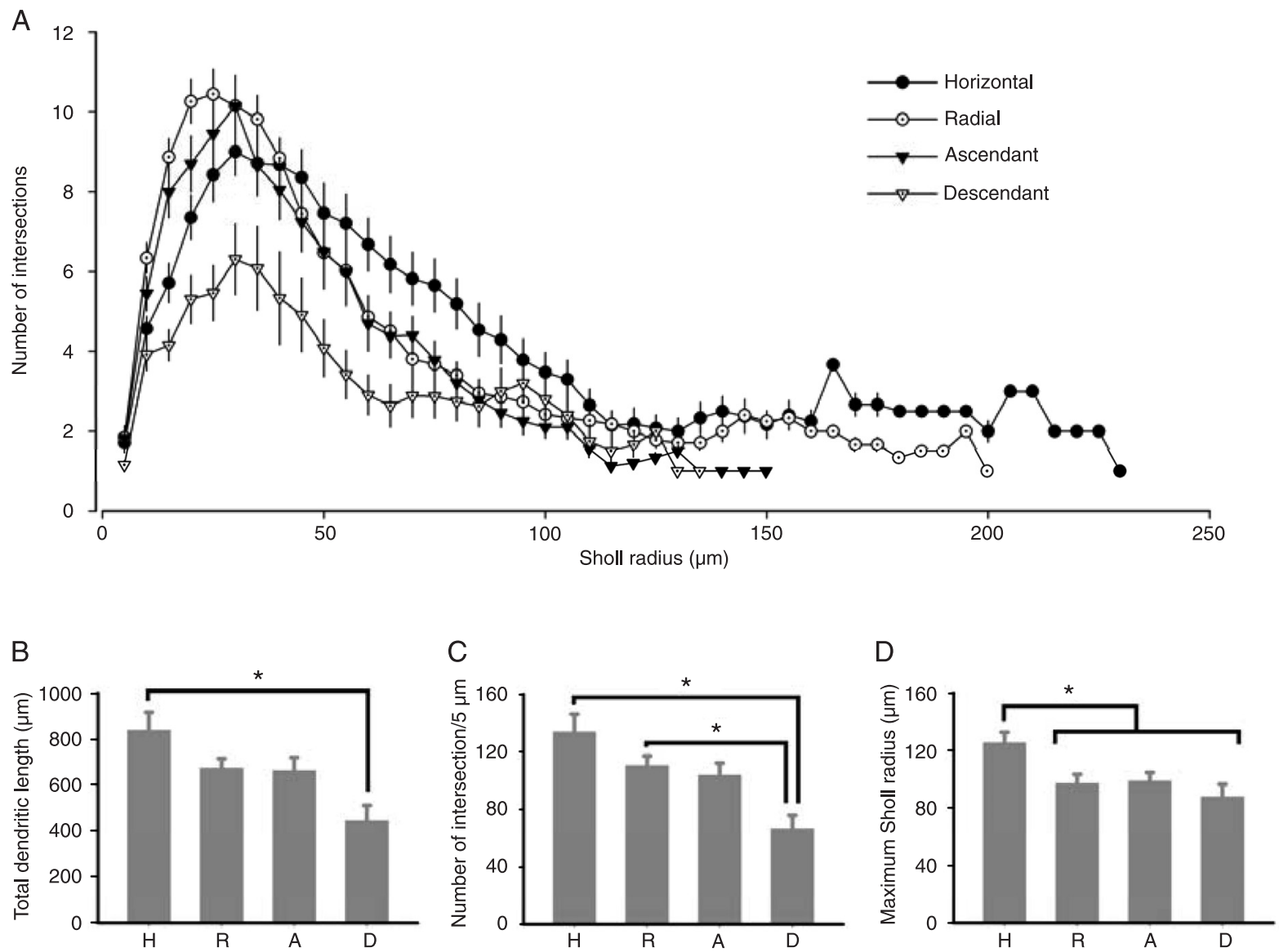

Figure 5. Morphological properties of labeled layer 1 neurons. Measurements were made for all horizontal $(H ; N=28)$, radial $(R ; N$ $=41)$, ascendant $(A ; N=22)$, and descendant $(D ; N=13)$ cells. $A$, Sholl analysis of each morphological subtype. $B$, Total dendritic length of each morphotype. Note the significant difference between horizontal and descendant cells. $C$, Number of intersections per 5 $\mu \mathrm{m}$ for each morphotype. Note that descendant cells differ from horizontal and radial neurons regarding this parameter, but horizontal and radial cells do not differ between each other. $D$, Maximum Sholl radius for each morphotype. Note that the horizontal cells differ significantly from all remaining populations. ${ }^{*} \mathrm{P}<0.05$, Kruskal-Wallis ANOVA. 
and maximum Sholl radius between morphotypes (Figure $5 B-D)$. Descendant cells had consistently smaller total dendritic lengths than horizontal cells and a lower number of intersections per $5 \mu \mathrm{m}$ than horizontal and radial neurons (Figure 5C). Horizontal cells had a larger maximum Sholl radius than all other subtypes (Figure 5D).

For the analysis of the spatial distribution of layer 1 neurons, we divided the layer into three equally sized sublayers (superior, medial, and inferior sublayers) according to their distance from the pial surface and the border with cortical layer $2 / 3$ (1). The distribution of morphologically distinct populations across these sub-lamina of layer 1 was highly unequal $(P<0.01)$, with the descendant cells showing a highly different spatial organization in relation to the remaining subtypes. Descendant neurons were especially concentrated in the superior sublayer $(76.9 \%$ of all descendant cells; 10/13), relatively rare in the medial sublayer $(23.1 \% ; 3 / 13)$ and were totally absent from the inferior sublayer.

The distribution of the remaining morphotypes was statistically similar, with most non-descendant cells being concentrated in the medial sublayer $(\approx 50 \%$ of all nondescendant cells; 44/92) and being common in the inferior one ( $\approx 30-40 \%$; 34/92). Approximately $10-20 \%$ (14/92) of non-descendant cells were located in the superior sublayer. Horizontal, ascendant and radial cells thus made up the whole population of the inferior sublayer, as no descendant cells where found in this stratum.

Electrophysiological parameters of recorded layer 1 interneurons are summarized in Tables 1, 2, and 3. Sponta- neous action potentials were rare and were not investigated. Passive membrane properties and the firing frequency of each morphotype and sublayer can be seen in Figure 6. These parameters were not significantly different among

Table 1. Summary of electrophysiological parameters in relation to morphological classification.

\begin{tabular}{|c|c|c|c|c|}
\hline \multirow[t]{2}{*}{ Parameters } & \multicolumn{4}{|c|}{ Morphological classification } \\
\hline & Horizontal & Radial & Ascending & Descending \\
\hline Number of cells & 28 & 41 & 22 & 13 \\
\hline $\mathrm{RMP}(\mathrm{mV})$ & $-54.23 \pm 1.18$ & $-56.09 \pm 1.07$ & $-55.50 \pm 1.17$ & $-53.57 \pm 1.45$ \\
\hline AP threshold (mV) & $-40.72 \pm 0.94$ & $-41.31 \pm 0.45$ & $-41.65 \pm 0.75$ & $-41.09 \pm 1.11$ \\
\hline $\mathrm{R}_{\text {in }}(\mathrm{M} \Omega)$ & $539.61 \pm 46.69$ & $512.91 \pm 41.23$ & $526.47 \pm 34.95$ & $505.96 \pm 65.02$ \\
\hline Spike frequency $(\mathrm{Hz})$ & $29.96 \pm 2.25$ & $30.23 \pm 2.71$ & $38.33 \pm 4.05$ & $33.99 \pm 3.58$ \\
\hline AP amplitude (mV) & $93.25 \pm 2.73$ & $97.47 \pm 1.67$ & $94.83 \pm 2.68$ & $95.85 \pm 2.09$ \\
\hline Half width (ms) & $1.09 \pm 0.04$ & $0.94 \pm 0.03$ & $0.96 \pm 0.03$ & $0.92 \pm 0.05$ \\
\hline $10-90 \%$ rise time $(\mathrm{ms})$ & $0.44 \pm 0.02$ & $0.36 \pm 0.01$ & $0.38 \pm 0.01$ & $0.38 \pm 0.02$ \\
\hline $10-90 \%$ decay time (ms) & $0.74 \pm 0.04$ & $0.65 \pm 0.03$ & $0.63 \pm 0.03$ & $0.60 \pm 0.05$ \\
\hline$\tau(\mathrm{ms})$ & $51.38 \pm 4.29$ & $47.89 \pm 4.52$ & $51.40 \pm 4.91$ & $44.27 \pm 8.39$ \\
\hline fAHP $(m V)$ & $15.88 \pm 2.26$ & $16.84 \pm 1.77$ & $13.40 \pm 13.40$ & $16.33 \pm 5.86$ \\
\hline mAHP (mV) & $14.81 \pm 1.90$ & $17.08 \pm 1.09$ & $13.46 \pm 1.55$ & $9.47 \pm 4.95$ \\
\hline $\mathrm{sAHP}(\mathrm{mV})$ & $7.73 \pm 0.58$ & $7.68 \pm 1.08$ & $5.90 \pm 1.17$ & $5.17 \pm 1.98$ \\
\hline ADP (mV) & $2.67 \pm 2.41$ & $3.25 \pm 1.80$ & - & - \\
\hline
\end{tabular}

Data are reported as means \pm SEM. RMP = resting membrane potential; $A P=$ action potential; $\mathrm{R}_{\text {in }}=$ input resistance; $\tau=$ membrane time constant; fAHP = fast after-hyperpolarization potentials; $\mathrm{mAHP}=$ medium after-hyperpolarization potentials; sAHP = slow after-hyperpolarization potentials; ADP = after-depolarization potentials.

Table 2. Summary of electrophysiological parameters in relation to spatial distribution.

\begin{tabular}{lcccc}
\hline \multirow{2}{*}{ Parameters } & \multicolumn{4}{c}{ Spatial distribution } \\
\cline { 2 - 5 } & Whole layer 1 & Superior & Medial & Inferior \\
\hline Number of cells & 105 & 24 & 47 & 34 \\
RMP $(\mathrm{mV})$ & $-56.64 \pm 6.32$ & $-55.45 \pm 7.14$ & $-54.86 \pm 5.65$ & $-56.11 \pm 5.16$ \\
AP threshold $(\mathrm{mV})$ & $-41.52 \pm 6.21$ & $-40.27 \pm 4.71$ & $-41.09 \pm 2.83$ & $-42.31 \pm 2.61$ \\
Rin $(\mathrm{M} \Omega)$ & $500.25 \pm 197.62$ & $509.36 \pm 191.27$ & $515.35 \pm 220.75$ & $530.50 \pm 203.58$ \\
Spike frequency $(\mathrm{Hz})$ & $30.99 \pm 14.03$ & $32.51 \pm 10.05$ & $34.14 \pm 17.24$ & $31.05 \pm 13.00$ \\
AP amplitude $(\mathrm{mV})$ & $96.31 \pm 10.99$ & $93.88 \pm 9.88$ & $92.71 \pm 9.84$ & $99.77 \pm 12.12$ \\
Half width $(\mathrm{ms})$ & $0.94 \pm 0.20$ & $0.94 \pm 0.19$ & $0.94 \pm 0.21$ & $1.02 \pm 0.21$ \\
$10-90 \%$ rise time $(\mathrm{ms})$ & $0.37 \pm 0.09$ & $0.37 \pm 0.08$ & $0.37 \pm 0.08$ & $0.41 \pm 0.09$ \\
$10-90 \%$ decay time $(\mathrm{ms})$ & $0.63 \pm 0.16$ & $0.62 \pm 0.14$ & $0.64 \pm 0.19$ & $0.70 \pm 0.17$ \\
$\tau(\mathrm{ms})$ & $43.66 \pm 20.43$ & $45.01 \pm 23.38$ & $45.63 \pm 22.37$ & $47.74 \pm 21.82$ \\
fAHP $(\mathrm{mV})$ & $17.48 \pm 6.05$ & $18.89 \pm 5.30$ & $20.38 \pm 6.38$ & $13.91 \pm 3.62$ \\
mAHP $(\mathrm{mV})$ & $14.36 \pm 5.38$ & $17.41 \pm 5.32$ & $16.89 \pm 4.79$ & $12.22 \pm 5.73$ \\
sAHP $(\mathrm{mV})$ & $5.42 \pm 3.55$ & $6.90 \pm 5.31$ & $6.68 \pm 3.45$ & $5.31 \pm 2.49$ \\
ADP $(\mathrm{mV})$ & $4.90 \pm 3.90$ & $6.53 \pm 3.11$ & $7.00 \pm 2.70$ & $3.46 \pm 3.38$ \\
\hline
\end{tabular}

Data are reported as means \pm SEM. RMP = resting membrane potential; AP = action potential; $\mathrm{R}_{\text {in }}=$ input resistance; $\tau=$ membrane time constant; fAHP = fast after-hyperpolarization potentials; $\mathrm{mAHP}=$ medium after-hyperpolarization potentials; $\mathrm{sAHP}=$ slow after-hyperpolarization potentials; ADP = after-depolarization potentials. 
the studied subdivisions of layer 1 neurons $(P>0.05)$.

Of the 244 recorded cells, 219 were submitted to successive depolarizing pulses and their active properties were investigated. All recorded cells were capable of repetitive firing, with variable frequencies. Of these 219 studied neurons, $93.61 \%(205 / 219)$ showed little or no frequency adaptation; $59.82 \%(131 / 219)$ of neurons presented fAHPs, $72.15 \%$ (158/219) presented mAHPs, $76.71 \%$ (168/219) presented sAHPs, and $74.20 \%(53 / 219)$ exhibited ADPs. It should be noted that we did not observe co-occurrence of an mAHP and SAHP in a single cell during our experiments, which is in agreement with previous studies by Zhou and Hablitz (2). The mean amplitudes of these after-potentials are presented in Tables 1, 2, and 3. Representative recordings of each type of after-potential are shown in Figure 7.

Action potential waveforms were defined and categorized according to the parameters used by Zhou and Hablitz (2). According to these criteria, most of the recorded neurons (162/219) could be identified as classic non-accommodating cells (c-NAC) (Figure 8A), i.e., cells with short-duration action potentials (around $1.1 \mathrm{~ms}$ ), rapid

Table 3. Summary of electrophysiological parameters in relation to neuronal firing pattern.

\begin{tabular}{lcccc}
\hline \multirow{2}{*}{ Parameters } & \multicolumn{4}{c}{ Neuronal firing pattern } \\
\cline { 2 - 5 } & c-NAC & c-AC & c-STUT & d-NAC \\
\hline Number of cells & 162 & 35 & 10 & 29 \\
RMP $(\mathrm{mV})$ & $-56.15 \pm 0.51$ & $-55.49 \pm 0.91$ & $-63.40 \pm 1.95$ & $-58.31 \pm 0.95$ \\
AP threshold $(\mathrm{mV})$ & $-41.75 \pm 0.26$ & $-41.01 \pm 0.46$ & $-40.44 \pm 0.66$ & $-42.28 \pm 0.50$ \\
$\mathrm{R}_{\text {in }}(\mathrm{M} \Omega)$ & $538.12 \pm 17.18$ & $428.41 \pm 19.29$ & $331.83 \pm 32.37$ & $442.67 \pm 22.27$ \\
Spike frequency $(\mathrm{Hz})$ & $33.21 \pm 1.23$ & $27.31 \pm 1.43$ & $18.48 \pm 3.24$ & $26.10 \pm 1.70$ \\
AP amplitude $(\mathrm{mV})$ & $96.10 \pm 0.90$ & $99.46 \pm 1.45$ & $90.46 \pm 2.97$ & $99.89 \pm 1.80$ \\
Half width (ms) & $0.94 \pm 0.02$ & $0.93 \pm 0.03$ & $0.85 \pm 0.05$ & $0.91 \pm 0.03$ \\
10-90\% rise time $(\mathrm{ms})$ & $0.37 \pm 0.01$ & $0.37 \pm 0.01$ & $0.33 \pm 0.02$ & $0.36 \pm 0.01$ \\
10-90\% decay time $(\mathrm{ms})$ & $0.64 \pm 0.01$ & $0.62 \pm 0.02$ & $0.55 \pm 0.04$ & $0.62 \pm 0.03$ \\
$\tau(\mathrm{ms})$ & $46.20 \pm 1.66$ & $38.77 \pm 2.93$ & $23.96 \pm 3.64$ & $42.79 \pm 3.65$ \\
fAHP $(\mathrm{mV})$ & $16.98 \pm 0.52$ & $21.33 \pm 1.11$ & $16.17 \pm 0.09$ & $16.71 \pm 0.80$ \\
mAHP $(\mathrm{mV})$ & $12.77 \pm 0.42$ & $16.90 \pm 0.85$ & $19.28 \pm 1.17$ & $15.50 \pm 0.93$ \\
sAHP $(\mathrm{mV})$ & $5.19 \pm 0.29$ & $7.32 \pm 0.67$ & $6.23 \pm 0.40$ & $4.17 \pm 0.48$ \\
ADP $(\mathrm{mV})$ & $5.63 \pm 0.32$ & $5.58 \pm 0.83$ & $2.13 \pm 0.48$ & $2.51 \pm 0.31$ \\
\hline
\end{tabular}

Data are reported as means \pm SEM. c-NAC and c-AC = classic non-accommodating and accommodating cells, respectively; c-STUT = classic stuttering neurons; $d-N A C=$ delayed NAC; RMP $=$ resting membrane potential; $\mathrm{AP}=$ action potential; $\mathrm{R}_{\text {in }}=$ input resistance; $\tau$ = membrane time constant; $\mathrm{fAHP}=$ fast after-hyperpolarization potentials; $\mathrm{mAHP}=$ medium after-hyperpolarization potentials; sAHP = slow after-hyperpolarization potentials; ADP $=$ after-depolarization potentials.

A
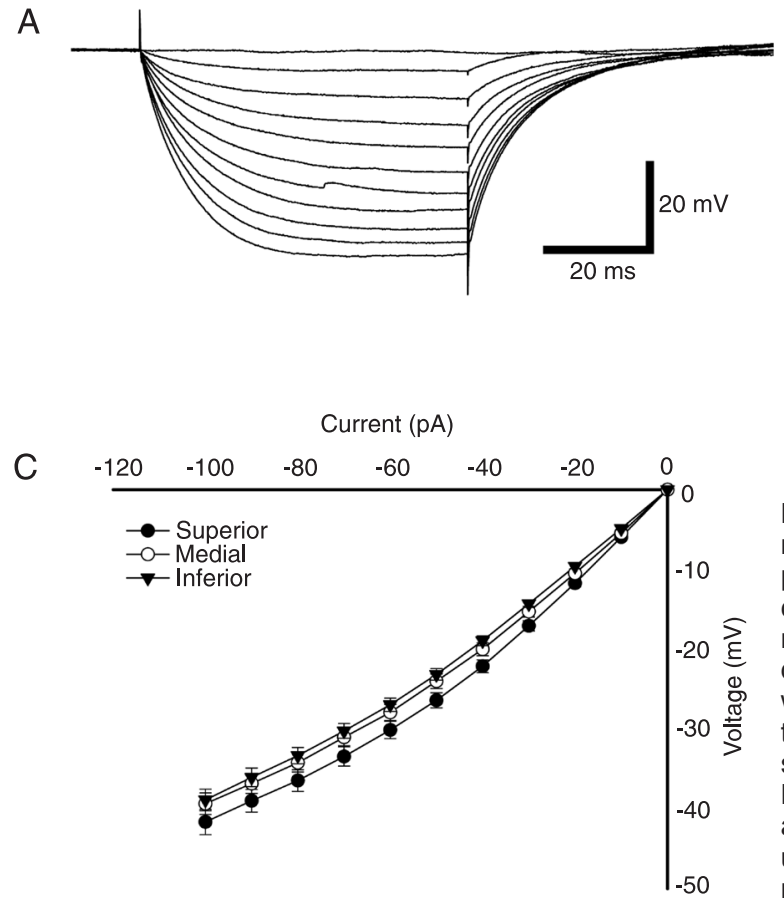

B

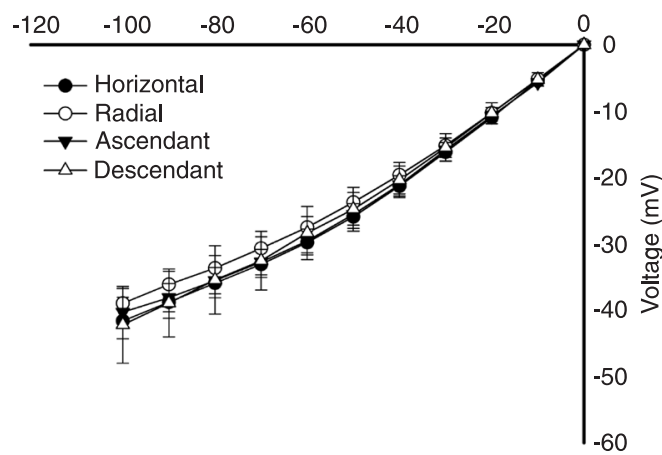

Figure 6. Passive electrophysiological properties of layer 1 interneurons. $A$, Representative recording of a neuron under successive hyperpolarizing pulses (varying in $10 \mathrm{pA}$ steps). B, Current-voltage (I/N) curve of layer 1 neurons according to dendritic morphotype. Measurements were made for all horizontal $(\mathrm{N}=28)$, radial $(\mathrm{N}=41)$, ascendant $(N=22)$, and descendant $(N=13)$ cells. Points were calculated with the mean values obtained for all studied cells in each group. Note that there was no statistically significant segregation between the data sets. $C, \mathrm{I} / \mathrm{V}$ curve of layer 1 neurons according to sublayer distribution. Measurements were made for all superior $(N=24)$, medial $(N=47)$ and inferior $(\mathrm{N}=34)$ cells. Points were calculated with the mean values obtained for all cells studied in each group. Note that there was no statistically significant differences between the data sets. 

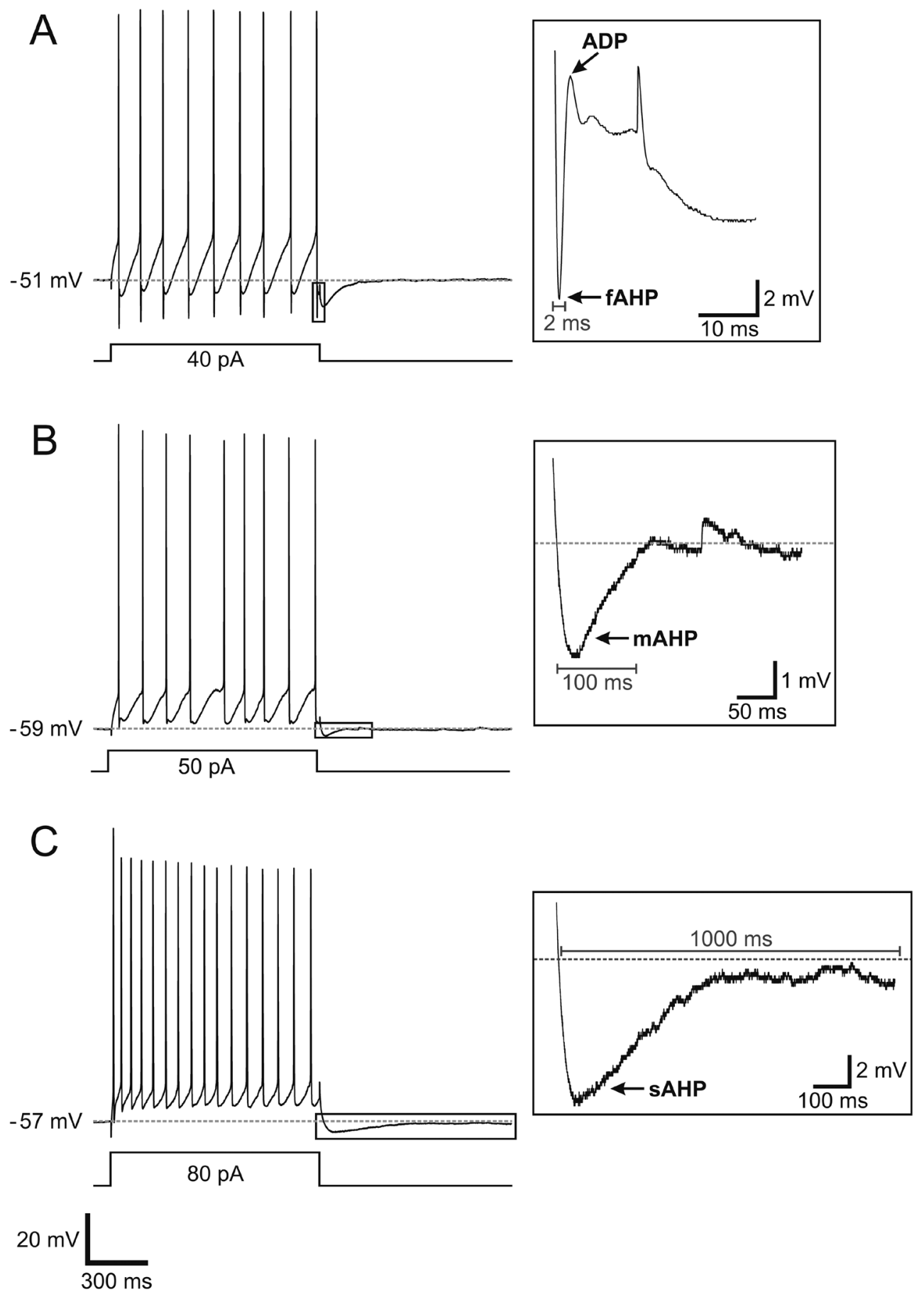

Figure 7. Afterpotentials of layer 1 interneurons. Each insert represents an expanded view of the area delimited by the black squares in the representative electrophysiological recordings. Gray lines represent the membrane threshold. Gray scales represent the width range used to identify each type of after-potential, according to the established criteria described by Storm (18). A, Representative recording of a layer 1 neuron submitted to a depolarizing current pulse and the detail of a fast hyperpolarization (fAHP) with an accompanying after-depolarization potential (ADP). $B$, Representative recording of a layer 1 neuron submitted to a depolarizing current pulse and the detail of a medium hyperpolarization (mAHP). C, Representative recording of a layer 1 neuron submitted to a depolarizing current pulse and the detail of a slow hyperpolarization (sAHP). 

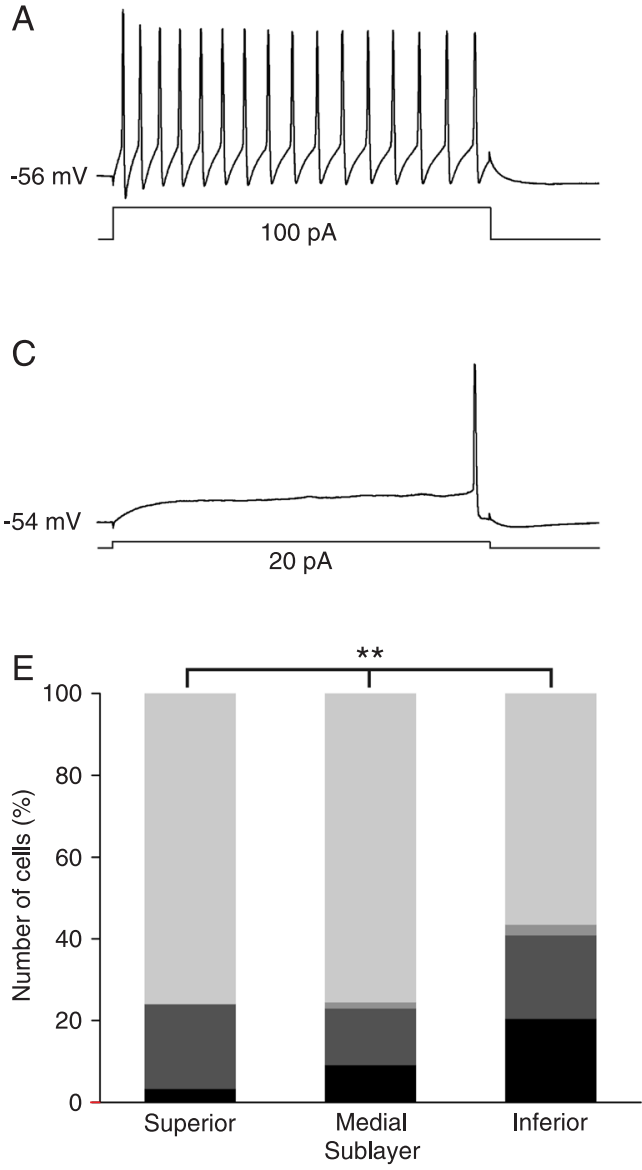
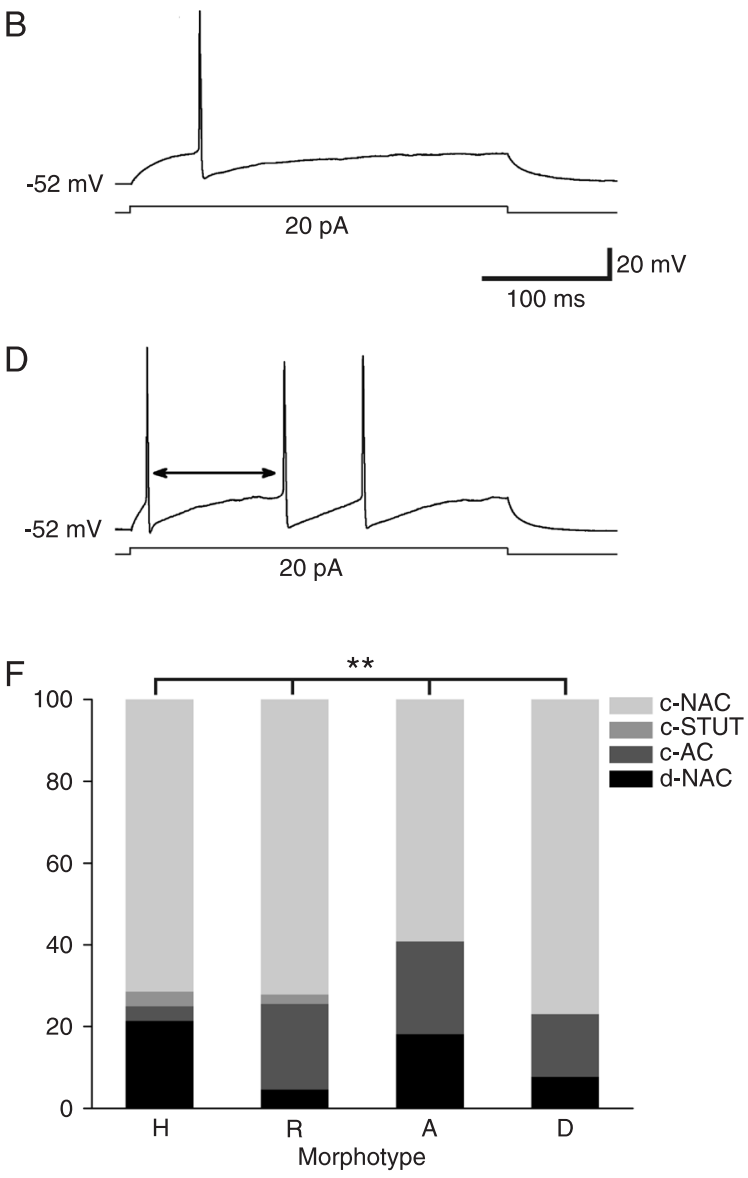

Figure 8. Intrinsic firing patterns of layer 1 neurons. Recordings are representative of the general behavior of each cell type. A, Representative recording of a c-NAC cell (100 pA). B, Example of a c-AC cell (20 pA). C, Recording of a d-NAC cell $(20 \mathrm{pA})$. $D$, Example of a layer $1 \mathrm{c}$-STUT cell $(20 \mathrm{pA})$. The arrow indicates the duration of the quiescent period. $E$, Distribution of the proportion of firing patterns among the different morphotypes found in layer 1 . $F$, Distribution of the proportion of firing patterns among the different sublayers of layer $1 . \mathrm{H}=$ horizontal; $\mathrm{R}=$ radial; $\mathrm{A}=$ ascendant; $\mathrm{D}=$ descendant. c-NAC = classic non-accommodating; $c-S T U T=$ classic stuttering; $c-A C=$ classic accommodating; $d-N A C$ $=$ delayed non-accommodating. ${ }^{* *} \mathrm{P}<0.01$, G-test.

rate of spike repolarization with the presence of fAHPs and absence or near-absence of spike-frequency adaptation. These cells responded immediately and continued to fire during the entire duration of each stimulus (Figure 8A). They had a significantly higher $\mathrm{R}_{\mathrm{in}}$ and lower mAHP amplitude (Table 3; $\mathrm{P}<0.05$ ) than other recorded neurons in our study. This finding is similar to previous results about layer 1 neurons reported by Zhou and Hablitz (2). It should be noted that this C-NAC waveform is exclusive to cortical inhibitory interneurons $(12,26)$.

In spite of this uniformity in the action potential waveform, we found that the firing pattern of the studied cells showed marked firing pattern subtypes. Of the recorded neuronal population, $13.24 \%$ (29/219) of the cells were characterized as delayed non-accommodating neurons (d-NAC; Figure $8 \mathrm{C})$, i.e., cells that were not immediately responsive to a given depolarizing pulse, presenting spikes only after a certain period. These cells had significantly lower SAHP amplitudes (Table 3; $\mathrm{P}<0.05$ ). Another group of cells (15.98\%; 35/219) were classic accommodating neurons (c-AC; Figure 8B), in the sense that they responded with one or more action potentials (depending on the amplitude of the stimulus) and then silenced for the remainder of the pulse. These cells had a significantly higher fAHP amplitude (Table 3; $P<0.05)$. A fraction of $4.57 \%$ (10/219) was classified as classic stuttering cells (c-STUT; presenting quiescent periods, i.e., periods of intermittent silencing in their repetitive firing sequence; Figure 8D). These cells had a significantly higher RMP and lower $\tau$ and $R_{\text {in }}$ (Table 3; $P<0.05)$. Similar patterns were also found by Zhou and Hablitz (2) and Chu et al. (4).

A significant difference was found in the distribution 
of firing patterns between the distinct morphotypes $(P<$ 0.001). Ascendant and descendant cells did not exhibit a c-NAC firing pattern and horizontal cells presented a much smaller proportion of c-AC patterns (Figure 8F). In addition, ascendant and descendant cells, in marked contrast to the other morphological categories, did not exhibit ADPs. There was also a significant difference between the neuronal populations of each sublayer $(P=0.017)$. Cells of the superior sublayer did not present c-NAC firing patterns and only a relatively small proportion of these neurons were d-NAC cells (Figure 8E). Additionally, a larger proportion of neurons in the inferior sublayer had a d-NAC firing pattern compared to medial cells. Interestingly, the remaining membrane properties, such as $\tau$, RMP and $R_{\text {in }}$ were statistically homogeneous $(P>0.05)$ among layer 1 cells of different morphotypes (Table 1 and Figure 6B) and sublayer distribution (Table 2 and Figure 6C).

\section{Discussion}

We applied biocytin labeling and patch-clamp recording methods to layer 1 interneurons in order to evaluate possible relationships between morphological and electrophysiological parameters in these cells. We also obtained data for the proposal of a quantitative, dendrite-based, morphological classification of rat layer 1 neurons. In contrast to previous studies by Zhou and Hablitz (5), we used a quantitative approach for the establishment of cell classes and investigated the morphology-electrophysiology relationship in layer 1 neurons using both categorical and continuous statistical analyses. To our knowledge, this is the first proposal of a quantitative analytical investigation of the functional correlates of dendritic morphology and spatial distribution in layer 1 interneurons.

We propose the division of layer 1 cells into four major groups according to the orientation of their dendritic arborization clusters: horizontal, radial, ascendant, and descendant. According to our results, radial and ascendant cells are relatively uniform in their morphology. Horizontal cells, on the other hand, can be further segregated into cells that extend their dendrites to both sides of the soma and those that project unilaterally. Descendant cells can be divided into regular descendant cells, which could be described as "inverted" ascendant cells, and the subpial cells, a relatively rare subpopulation that was restricted to the superior sublayer of layer 1.

The absence of Cajal-Retzius cells is explained by the age of the animals studied (14 to 21 postnatal days), as the number of these cells decreases rapidly after 8 postnatal days (25). Insightful studies on the function of these cells can be found elsewhere $(2,5,8,9,25)$.

Horizontal, ascendant and radial neurons had a similar distribution across the established layer 1 subdivisions. Descendant cells, on the other hand, had a very distinct spatial organization, being almost restricted to the out- ermost region of layer 1 . We were unable to establish a physiological explanation for such a unique distribution. However, considering the distribution of axonal fibers in layer 1 (1), specifically the fact that the sublayer closest to the pia-mater typically contains few, poorly myelinated axons and that the medial sublayer contains many, highly myelinated fibers, we hypothesize that descendant cells, especially the subpial cells, are probably primarily involved in local intralayer circuits, while the remaining subtypes (concentrated on the medial and inferior sub-lamina), may be directly involved in interlayer and extracortical connections. Future studies, perhaps using retrograde tracing methods, must be done to confirm this hypothesis. Special attention should also be given to the rare and distinct subpial cells, whose highly distinct morphological properties hint at the possibility that they may execute a unique function in layer 1 physiology.

Due to their significantly large total dendritic length and maximum Sholl radius, horizontal cells may perhaps serve an integrative function, computing inputs over a relatively large receptor field. The probable role of radial and ascendant cells is harder to evaluate and more research is needed before any substantial hypothesis can be proposed.

The overall values of passive and active electrophysiological properties of layer 1 neurons were similar to those found in previous studies (2,9). However, a significant difference was found in the distribution of firing patterns between the established morphotypes and between the neuronal populations of each sublayer. To our knowledge, this is the first report of such relationships in layer 1 neurons. Even though the high variability of the dendritic organization of cortical inhibitory interneurons has led some authors to remark that only the analysis of axonal arborization could provide a solid distinction between the physiological roles of cortical interneurons (12), our data show that there are clear differences in the statistical distribution of firing patterns between layer 1 neurons with distinct dendritic morphological phenotypes and spatial distribution.

On the basis of our results, one could hypothesize that reported functional electrophysiological differences in layer 1 neurons, such as between d-NAC and non-d-NAC cells in the integration of sensory input (4), may also be correlated to certain morphological parameters. In this context, we believe that the different distribution of firing properties between the proposed morphotypes is probably related to differences in the computational or functional roles of each structurally defined population in layer 1 circuits. Each pattern of dendritic distribution could perhaps be related to different patterns of synaptic input during cortical maturation and/or early functional processing of sensory phenomena. The observed differences in firing patterns among the substrata of layer 1 could be related to differences in the nature of the synaptic input arriving from distinct types of afferent subcortical fibers, as previously cited (1). In addition, ascendant and descendant cells, in marked contrast to the other 
morphological categories, did not exhibitADPs. These variations in electrophysiological parameters of layer 1 neurons are probably due to the differential expression, insertion or deactivation of specific ion channels, which in turn could be related to genetic or ontological differences among different morphological subtypes or spatially segregated neuronal populations in this cortical layer $(12,26,27)$.

Our results show that dendritic morphology and spatial distribution of layer 1 interneurons, parameters that have been overlooked by previous studies on this neuronal population, are correlated with important distinctions in neuronal firing pattern. Given the amount of hypotheses that arise from these findings, further studies must be done in order to fully understand the origins and functional implications of the relationships between dendritic morphology, spatial distribution and physiological parameters.

\section{References}

1. Vogt BA. The role of layer I in cortical function. In: Peters A, Jones E (Editors), Cerebral cortex. New York: Plenum; 1991.

2. Zhou FM, Hablitz JJ. Layer I neurons of rat neocortex. I. Action potential and repetitive firing properties. $J$ Neurophysiol 1996; 76: 651-667.

3. Shlosberg D, Patrick SL, Buskila Y, Amitai Y. Inhibitory effect of mouse neocortex layer I on the underlying cellular network. Eur J Neurosci 2003; 18: 2751-2759.

4. Chu Z, Galarreta M, Hestrin S. Synaptic interactions of latespiking neocortical neurons in layer 1 . J Neurosci 2003; 23: 96-102.

5. Zhou FM, Hablitz JJ. Morphological properties of intracellularly labeled layer I neurons in rat neocortex. J Comp Neurol 1996; 376: 198-213.

6. Llinas RR, Leznik E, Urbano FJ. Temporal binding via cortical coincidence detection of specific and nonspecific thalamocortical inputs: a voltage-dependent dye-imaging study in mouse brain slices. Proc Natl Acad Sci U S A 2002; 99: 449-454.

7. Zhu Y, Zhu JJ. Rapid arrival and integration of ascending sensory information in layer 1 nonpyramidal neurons and tuft dendrites of layer 5 pyramidal neurons of the neocortex. $J$ Neurosci 2004; 24: 1272-1279.

8. Kirmse K, Dvorzhak A, Grantyn R, Kirischuk S. Developmental downregulation of excitatory GABAergic transmission in neocortical layer I via presynaptic adenosine A(1) receptors. Cereb Cortex 2008; 18: 424-432.

9. Zhou FM, Hablitz JJ. Layer I neurons of the rat neocortex. II. Voltage-dependent outward currents. J Neurophysiol 1996; 76: 668-682.

10. Zhou FM, Hablitz JJ. Rapid kinetics and inward rectification of miniature EPSCs in layer I neurons of rat neocortex. $J$ Neurophysiol 1997; 77: 2416-2426.

11. Hestrin S, Armstrong WE. Morphology and physiology of cortical neurons in layer I. J Neurosci 1996; 16: 5290-5300.

12. Markram H, Toledo-Rodriguez M, Wang Y, Gupta A, Silberberg G, Wu C. Interneurons of the neocortical inhibitory system. Nat Rev Neurosci 2004; 5: 793-807.

\section{Acknowledgments}

The authors are grateful to Dr. Marco Antonio V. Santos and the Evandro Chagas Institute (Ananindeua, Pará, Brazil) for providing the animals used in this study. We also wish to thank Prof. Dr. Jessé C. Costa for technical support in elaborating the custom Matlab scripts. Research supported by CNPq, FINEP, and the Foundation for Research Support of the State of Pará (FAPESPA). More specifically, we received the CNPq-PRONEX/FAPESPA (\#2268), and CNPq (\#470827/2009-2) research grants. D.V.V. Santos received a fellowship for graduate students from CAPES. K.M. Costa and M.C.G. Vaz received CNPq fellowships for undergraduate students. M. Da Silva Filho is currently a CNPq research fellow.
13. Shepherd GM. Electrotonic properties of axons and dendrites. In: Squire LR, Berg D, Bloom FE, du-Lac S, Ghosh A, Spitzer NC (Editors), Fundamental neuroscience. 3rd edn. London: Academic Press; 2008. p 87-108.

14. London M, Hausser M. Dendritic computation. Annu Rev Neurosci 2005; 28: 503-532.

15. Rall W. Core conducter theory and cable properties in neurons. In: Kandel ER, Mountcastle VB (Editors), Handbook of physiology: The nervous system. Bethesda: American Physiological Society; 1977. p 39-97.

16. Edwards FA, Konnerth A, Sakmann B, Takahashi T. A thin slice preparation for patch clamp recordings from neurones of the mammalian central nervous system. Pflugers Arch 1989; 414: 600-612.

17. Horikawa K, Armstrong WE. A versatile means of intracellular labeling: injection of biocytin and its detection with avidin conjugates. J Neurosci Methods 1988; 25: 1-11.

18. Storm JF. Intracellular injection of a $\mathrm{Ca}^{2+}$ chelator inhibits spike repolarization in hippocampal neurons. Brain Res 1987; 435: 387-392.

19. Meijering E, Jacob M, Sarria JC, Steiner $P$, Hirling $H$, Unser $M$. Design and validation of a tool for neurite tracing and analysis in fluorescence microscopy images. Cytometry $A$ 2004; 58: 167-176.

20. Hamam BN, Amaral DG, Alonso AA. Morphological and electrophysiological characteristics of layer $\mathrm{V}$ neurons of the rat lateral entorhinal cortex. J Comp Neurol 2002; 451: 45-61.

21. Romanova EV, Fosser KA, Rubakhin SS, Nuzzo RG, Sweedler JV. Engineering the morphology and electrophysiological parameters of cultured neurons by microfluidic surface patterning. FASEB J 2004; 18: 1267-1269.

22. Sholl DA. Dendritic organization in the neurons of the visual and motor cortices of the cat. $J$ Anat 1953; 87: 387-406.

23. Watson KK, Jones TK, Allman JM. Dendritic architecture of the von Economo neurons. Neuroscience 2006; 141: 11071112.

24. Ghosh A. Sholl Analysis Plugin (v1.0). http://biology.ucsd. edu/labs/ghosh/software/. Accessed November 10, 2010.

25. Bradford R, Parnavelas JG, Lieberman AR. Neurons in layer 
I of the developing occipital cortex of the rat. J Comp Neurol 1977; 176: 121-132.

26. Descalzo VF, Nowak LG, Brumberg JC, McCormick DA, Sanchez-Vives MV. Slow adaptation in fast-spiking neurons of visual cortex. J Neurophysiol 2005; 93: 1111-1118.

27. Budde T, White JA. The voltage-dependent conductances of rat neocortical layer I neurons. Eur J Neurosci 1998; 10: 2309-2321. 